\title{
Four New Lignans from Viburnum foetidum var. foedidum
}

\author{
Xuan-Qin Chen, ${ }^{a, b}$ Yan Li, ${ }^{a}$ Juan He,${ }^{a, b}$ Kou WAnG, ${ }^{a, b}$ Ming-Ming LI, ${ }^{a, b}$ Zheng-Hong PAN, ${ }^{a, b}$ \\ Li-Yan Peng, ${ }^{a}$ Xiao Cheng, ${ }^{a}$ and Qin-Shi ZhaO ${ }^{*}, a$ \\ ${ }^{a}$ State Key Laboratory of Phytochemistry and Plant Resources in West China, Kunming Institute of Botany, Chinese \\ Academy of Sciences; Kunming, Yunnan 650204, P. R. China: and ${ }^{b}$ Graduate School of the Chinese Academy of Sciences, \\ Beijing 100039, P. R. China. Received April 30, 2009; accepted June 16, 2009; published online July 16, 2009
}

The $70 \%$ aqueous acetone extract of the aerial part of Viburnum foetidum var. foedidum afforded four new lignans (1-4) together with six known ones. The structures of the four new compounds were elucidated on the basis of 1D (dimensional), 2D-NMR, and mass spectral analysis. The structure of compound 1 , with a novel spirocyclic moiety, was also confirmed by X-ray diffraction analysis. Compounds $1-10$ were evaluated for their cytotoxic activity. However, none of the compounds showed significant cytotoxic activity.

Key words Viburnum foetidum var. foedidum; lignan; X-ray diffraction analysis

Plants of the Viburnum genus, which belong to the family Caprifliaceae, comprise about 230 species and are mainly distributed in Asia and South America. China has more than 70 species. ${ }^{1)}$ The use of Viburnum species in Chinese popular folk medicine has a long history. For example, the leaves and skins of $V$. cylindricum, called shuihongmu in China, are used for the treatment of cough, diarrhea, rheumatoid arthritis, and tumefaction. ${ }^{2} V$. odoratissimum, commonly known as shanhushu, is used for tumefaction and bone fractures. ${ }^{3)}$ Phytochemical studies revealed that Viburnum species mainly contain triterpenoids, ${ }^{4,5)}$ iridoids, ${ }^{6}$ vibsane-type diterpenes, ${ }^{7,8)}$ lignans, ${ }^{9)}$ and phenolic glycosides. ${ }^{10)}$ Vibsane-type diterpenes are an unusual group of natural products, which can be subdivided into three classes consisting of sevenmembered ring, eleven-membered ring, and rearranged types. ${ }^{1)}$ These diterpenoids have interesting biological activities such as piscicidal activity, plant growth regulatory activity, and cytotoxic activity. ${ }^{11,12}$ )

As a part of our search for new vibsane-type diterpenoids, the $70 \%$ aqueous acetone extract of the aerial part of the $V$. foetidum var. foedidum from Simao County, Yunnan Province, P. R. China, was investigated phytochemically. Four new lignans (1-4) together with six known ones were found. The isolation and structural elucidation of the four new lignans are reported here.

\section{Results and Discussion}

The $70 \%$ aqueous acetone extract of the aerial parts of $V$. foetidum var. foedidum was successively subjected to silica gel and Sephadex LH-20 column chromatography as well as semipreparative HPLC to afford 10 lignans (1-10). Compounds 5-10 were identified as prinoresinol (5), ${ }^{13)}$ syringaresinol (6), ${ }^{14)}$ olivil (7), ${ }^{15)}$ 4,4', $7,7^{\prime}$-tetrahydroxy-3,3' dimethoxy-9,9' -epoxylignan (8), ${ }^{16)} \quad 4,4^{\prime}, 8,9$-texahydroxy$3,3^{\prime}$-dimethoxy-7,9'-epoxylignan (9), ${ }^{17)}$ and padocin (10), ${ }^{18)}$ respectively, based on comparisons of their spectral data with those already reported.

Compound 1 was obtained as a white amorphous powder. Its molecular formula was established as $\mathrm{C}_{20} \mathrm{H}_{22} \mathrm{O}_{6}$ deduced from the HR-electrospray ionization (ESI)-MS at $\mathrm{m} / z$ $357.1330\left([\mathrm{M}-\mathrm{H}]^{-}\right)$and ${ }^{13} \mathrm{C}-\mathrm{NMR}$ data analysis, indicating 10 degrees of unsaturation. Its IR showed the presence of $\mathrm{OH}$ $\left(3536 \mathrm{~cm}^{-1}\right)$ and aromatic rings $\left(1611 \mathrm{~cm}^{-1}, 1596 \mathrm{~cm}^{-1}\right.$, and $\left.1520 \mathrm{~cm}^{-1}\right)$. The ${ }^{13} \mathrm{C}-\mathrm{NMR}$ and distortionless enhancement by polarization transfer (DEPT) spectra showed a total 20 of signals, which included two methines ( $\delta 46.5$ and 84.6), three methylenes ( $\delta 36.9,50.2$, and 72.3$)$, two methoxys $(2 \times \delta 56.4)$, one oxygenated $s p^{3}$ quaternary carbon $(\delta 71.4)$, and twelve other signals for two aromatic rings. In the ${ }^{1} \mathrm{H}$ NMR spectrum, three aromatic protons in an ABX pattern $[\delta$ $6.86(\mathrm{~d}, J=2.0 \mathrm{~Hz}), 6.79$ (d, $J=8.0 \mathrm{~Hz}), 6.73$ (dd, $J=2.0,8.0$ $\mathrm{Hz})]$ and a singlet for a methoxyl group $(\delta 3.80)$ indicated the existence of a 3-hydroxy-4-methoxyphenyl ring. Three additional aromatic protons in another ABX pattern $[\delta 6.33$ $(\mathrm{d}, J=2.0 \mathrm{~Hz}), 6.81(\mathrm{~d}, J=8.0 \mathrm{~Hz}), 6.59(\mathrm{dd}, J=2.0,8.0 \mathrm{~Hz})]$ and a singlet for the other methoxyl group $(\delta 3.85)$ indicated the existence of a 4-hydroxy-3-methoxyphenyl ring. The NMR data of $\mathbf{1}$ were closely similar to those of lariciresinol. ${ }^{13)}$ The clearest differences between compound $\mathbf{1}$ and lariciresinol were less $\mathrm{CH}$, a more oxygenated quaternary carbon ( $\delta 71.4)$, and an obvious upfield shift of C-9 in 1. Considering the characteristic NMR spectrum discussed above and the fact that $\mathbf{1}$ had an additional more ring system compared with that of lariciresinol, 1 was deduced to be 8,9epoxy-lariciresinol. The formation of 8,9-epoxy led to the upfield shift of C-9 and the presence of an oxygenated quaternary carbon $(\delta 71.4)$.

In the rotating frame Overhauser enhancement spectroscopy (ROESY) of 1 , the correlation of $\mathrm{H}-7$ with $\mathrm{H}-8^{\prime}$ indicated that both $\mathrm{H}-7$ and $\mathrm{H}-8^{\prime}$ were in the $\beta$-orientation. However, the ROESY spectrum could not provide sufficient information to elucidate the stereochemistry of C-8. After many attempts with different solvents, a single crystal of 1 was obtained in $\mathrm{MeOH}-\mathrm{H}_{2} \mathrm{O}(95: 5)$. A single crystal X-ray analysis clarified the relative configuration of $\mathrm{C}-8$ and unambiguously confirmed the structure of $\mathbf{1}$ (Fig. 1).

Compound 2, a white amorphous powder, had the molecular formula $\mathrm{C}_{20} \mathrm{H}_{23} \mathrm{O}_{6} \mathrm{Cl}$ based on the HR-ESI-MS at $\mathrm{m} / \mathrm{z}$ $417.1087\left([\mathrm{M}+\mathrm{Na}]^{+}\right)$. The isotopic ion peak pattern indicated the presence of one $\mathrm{Cl}$ atom. Its IR spectrum showed absorption bands typical of the hydroxyl group $\left(3523 \mathrm{~cm}^{-1}\right)$ and aromatic rings $\left(1602 \mathrm{~cm}^{-1}, 1592 \mathrm{~cm}^{-1}\right.$, and $\left.1516 \mathrm{~cm}^{-1}\right)$. The ${ }^{13} \mathrm{C}-\mathrm{NMR}$ and DEPT spectra showed two methines, three methylenes, one oxygenated $s p^{3}$ quaternary carbon, two methoxys, and twelve aromatic carbons. In the ${ }^{1} \mathrm{H}-\mathrm{NMR}$ spectrum, three singlet aromatic protons $[\delta 6.89(\mathrm{~s}), 6.81(\mathrm{~s})$, 


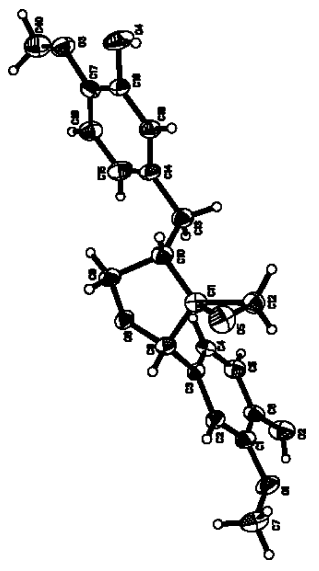

Fig. 1. Single X-Ray Diffraction of Compound 1

$6.80(\mathrm{~s})]$ together with the singlet for one methoxy $(\delta 3.77)$ indicated the existence of a 5-hydroxy-3-methoxyphenyl ring. Three other aromatic protons $[\delta 6.70(\mathrm{~d}, J=2.0), 6.72$ (d, $J=10.0$ ), 6.60 (dd, $J=2.0,10.0)]$ and the other methoxy $(\delta 3.80)$ were deduced due to the presence of a 4-hydroxy-3methoxyphenyl ring. Compounds $\mathbf{2}$ and $\mathbf{1}$ had the similar 1Dand 2D-NMR spectra. The most remarkable difference was the obvious downfield shift of C-8. Detailed analysis and comparison of the NMR data of $\mathbf{1}$ and $\mathbf{2}$ suggested that the 8,9-epoxy moiety in $\mathbf{1}$ has an additional $\mathrm{HCl}$ molecule. Chemical shifts of C-8 $(\delta 82.3)$ and C-9 $(\delta 48.8)$ indicated that the $\mathrm{OH}$ was attached to $\mathrm{C}-8$ and the $\mathrm{Cl}$ atom to $\mathrm{C}-9$. Therefore, 2 was established to be 5,4',8-trihydroxy-3,3'dimethoxy-8-chloromethylene-7,9'-epoxylignan. The relative configuration of $\mathbf{2}$ was determined on the basis of ROESY spectral analysis. ROSEY correlations of $\mathrm{H}-7$ with $\mathrm{H}-8^{\prime}$ and of $\mathrm{H}-8^{\prime}$ with $\mathrm{H} \beta-9$ indicated that $\mathrm{H}-7, \mathrm{H}-8^{\prime}$, and $-\mathrm{CH}_{2} \mathrm{Cl}$ were all in the $\beta$-orientation. Since $\mathrm{CHCl}_{3}$ was used during purification, 2 may have been an artifical compound. To determine whether $\mathbf{2}$ was a natural compound, the crude extract of this plant was analyzed in HPLC with $\mathbf{2}$ as an internal standard and the corresponding peak of $\mathbf{2}$ was clearly found in the crude extract, suggesting that $\mathbf{2}$ is a natural compound.

Compound 3 was obtained as a colorless gum. It had the molecular formula $\mathrm{C}_{21} \mathrm{H}_{26} \mathrm{O}_{6}$ according to its HR-ESI-MS at $m / z 397.1631\left([\mathrm{M}+\mathrm{Na}]^{+}\right)$. The IR spectrum revealed the presence of $\mathrm{OH}\left(3417 \mathrm{~cm}^{-1}\right)$ and aromatic rings $\left(1602 \mathrm{~cm}^{-1}\right.$, $1514 \mathrm{~cm}^{-1}$, and $\left.1434 \mathrm{~cm}^{-1}\right) .{ }^{13} \mathrm{C}$ - and DEPT NMR showed the presence of two aromatic rings, three methines (one bearing two oxygens at $\delta 105.5)$, three methylenes ( $\delta 33.5,38.9$, and 72.4), and three methoxys ( $\delta 54.6,55.9$, and 56.0). In its ${ }^{1} \mathrm{H}-\mathrm{NMR}$ spectrum, three aromatic protons $[\delta 6.73(\mathrm{~d}, J=2.0$ $\mathrm{Hz}), 6.75(\mathrm{~d}, J=10.0 \mathrm{~Hz}), 6.62(\mathrm{dd}, J=2.0,10.0 \mathrm{~Hz})]$ and three other aromatic protons $[\delta 6.70(\mathrm{~s}), 6.82(\mathrm{~d}, J=10.0 \mathrm{~Hz})$, $6.68(\mathrm{~d}, J=10.0 \mathrm{~Hz})]$ indicated the presence of two 1,3,4-substituted aromatic ring systems. In addition, three methoxy signals $(\delta 3.33,3.87,3.89)$ were also observed. The NMR data of 3 were similar to those of 4,4'-dihydroxy-3,3'dimethoxy-9-butoxy-9, $9^{\prime}$-epoxylignan. ${ }^{19)}$ The only significant difference was that the signal for butoxy was replaced by a methoxy ( $\delta 3.33)$. Therefore, 3 was inferred to be $4,4^{\prime}$ dihydroxy-3,3',9-trimethoxy-9,9'-epoxylignan, which can be further confirmed by heteronuclear multiple bond connectivity (HMBC) correlation from $\mathrm{H}-9$ to $\mathrm{C}-\mathrm{1}^{\prime \prime \prime \prime}$. The relative
Table $1 .{ }^{13} \mathrm{C}-\mathrm{NMR}$ Data of Compounds $\mathbf{1}-\mathbf{4}(125 \mathrm{MHz}, \delta$ in ppm)

\begin{tabular}{crrrr}
\hline \hline & \multicolumn{5}{c}{$\delta_{\mathrm{C}}$} \\
\cline { 2 - 5 } Position & \multicolumn{5}{c}{$\mathbf{1}^{a)}$} & $\mathbf{2}^{\text {b) }}$ & $\mathbf{3}^{b)}$ & $\mathbf{4}^{b)}$ \\
\cline { 2 - 5 } 1 & $131.2(\mathrm{~s})$ & $128.7(\mathrm{~s})$ & $133.0(\mathrm{~s})$ & $133.1(\mathrm{~s})$ \\
2 & $111.3(\mathrm{~d})$ & $109.0(\mathrm{~d})$ & $111.4(\mathrm{~d})$ & $112.7(\mathrm{~d})$ \\
3 & $147.6(\mathrm{~s})$ & $146.7(\mathrm{~s})$ & $145.0(\mathrm{~s})$ & $146.3(\mathrm{~s})$ \\
4 & $149.1(\mathrm{~s})$ & $114.5(\mathrm{~d})$ & $145.5(\mathrm{~s})$ & $146.8(\mathrm{~s})$ \\
5 & $116.2(\mathrm{~d})$ & $145.5(\mathrm{~s})$ & $114.2(\mathrm{~d})$ & $115.6(\mathrm{~d})$ \\
6 & $120.8(\mathrm{~d})$ & $119.0(\mathrm{~s})$ & $121.4(\mathrm{~d})$ & $123.0(\mathrm{~d})$ \\
7 & $84.5(\mathrm{~d})$ & $89.1(\mathrm{~d})$ & $33.5(\mathrm{t})$ & $40.2(\mathrm{t})$ \\
8 & $71.4(\mathrm{~s})$ & $82.3(\mathrm{~s})$ & $43.0(\mathrm{~d})$ & $47.4(\mathrm{~d})$ \\
9 & $50.2(\mathrm{t})$ & $48.8(\mathrm{t})$ & $105.5(\mathrm{~d})$ & $111.4(\mathrm{~d})$ \\
$1^{\prime}$ & $133.7(\mathrm{~s})$ & $132.7(\mathrm{~s})$ & $133.5(\mathrm{~s})$ & $135.3(\mathrm{~s})$ \\
$2^{\prime}$ & $116.8(\mathrm{~d})$ & $115.1(\mathrm{~d})$ & $114.8(\mathrm{~d})$ & $116.2(\mathrm{~d})$ \\
$3^{\prime}$ & $147.6(\mathrm{~s})$ & $145.3(\mathrm{~s})$ & $143.6(\mathrm{~s})$ & $145.4(\mathrm{~s})$ \\
$4^{\prime}$ & $147.6(\mathrm{~s})$ & $145.3(\mathrm{~s})$ & $146.2(\mathrm{~s})$ & $147.9(\mathrm{~s})$ \\
$5^{\prime}$ & $112.9(\mathrm{~d})$ & $120.0(\mathrm{~d})$ & $110.6(\mathrm{~d})$ & $112.0(\mathrm{~d})$ \\
$6^{\prime}$ & $121.0(\mathrm{~d})$ & $111.1(\mathrm{~d})$ & $120.0(\mathrm{~d})$ & $121.4(\mathrm{~d})$ \\
$7^{\prime}$ & $36.9(\mathrm{t})$ & $33.0(\mathrm{t})$ & $38.9(\mathrm{t})$ & $40.2(\mathrm{t})$ \\
$8^{\prime}$ & $46.5(\mathrm{~d})$ & $52.1(\mathrm{~d})$ & $52.2(\mathrm{~d})$ & $54.1(\mathrm{~d})$ \\
$9^{\prime}$ & $72.3(\mathrm{t})$ & $69.5(\mathrm{t})$ & $72.4(\mathrm{t})$ & $73.5(\mathrm{t})$ \\
$1^{\prime \prime} \mathrm{OCH}_{3}$ & $56.4(\mathrm{q})$ & $55.6(\mathrm{q})$ & $56.0(\mathrm{q})$ & $57.2(\mathrm{q})$ \\
$1^{\prime \prime \prime} \mathrm{OCH}_{3}$ & $56.4(\mathrm{q})$ & $55.7(\mathrm{q})$ & $55.9(\mathrm{q})$ & $57.4(\mathrm{q})$ \\
$1^{\prime \prime \prime \prime} \mathrm{OCH}_{3}$ & & & $54.6(\mathrm{q})$ & $56.2(\mathrm{q})$ \\
\hline & & & & \\
\hline
\end{tabular}

a) Recorded in $\mathrm{CD}_{3} \mathrm{OD}, b$ ) recorded in $\mathrm{CDCl}_{3}$.

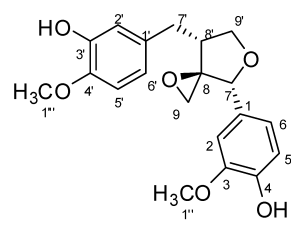<smiles>COc1cc(C=C2C(=O)OCC2Cc2ccc(O)c(OC)c2)ccc1O</smiles>

3<smiles>COc1cc(O)cc([C@@H]2OC[C@H](Cc3ccc(OC)c(O)c3)[C@H]2CCl)c1</smiles><smiles>COc1cc(C[C@@H]2C(OC)OC[C@@H]2Cc2ccc(O)c(OC)c2)ccc1O</smiles>

Fig. 2. Structures of Compounds $1-4$

stereochemistry of $\mathbf{3}$ was established on the basis of analysis of ROESY experiment and coupling constants. The ROESY correlation of H-9 with $\mathrm{H}-8^{\prime}$ and large coupling constant between $\mathrm{H}-9$ and $\mathrm{H}-8(J=5.0 \mathrm{~Hz})$ suggested that $\mathrm{H}-9, \mathrm{H}-8$, and $\mathrm{H}-8^{\prime}$ were in the $\beta-, \alpha$-, and $\beta$-orientation, respectively.

Compound 4 was obtained as a colorless gum. Although 4 and 3 presented a single spot on TLC (silica gel) developed in several solvent systems, they showed two close but separated peaks in HPLC analysis. Compound 4 was assigned the same molecular formula $\mathrm{C}_{21} \mathrm{H}_{26} \mathrm{O}_{6}$ as $\mathbf{3}$ on the basis of its HR-ESI-MS at $m / z 397.1636\left([\mathrm{M}+\mathrm{Na}]^{+}\right)$. Compound 4 exhibited similar UV, IR, and NMR spectra as $\mathbf{3}$ except for the different chemical shifts of C-7, C-8, and C-9. Detailed analysis of the 2D-NMR spectrum of $\mathbf{4}$ indicated that $\mathbf{4}$ and $\mathbf{3}$ had the same planar structure. The relative stereochemistry of 4 was established on the basis of analysis of the ROESY experiment and coupling constant. $\mathrm{H}-9, \mathrm{H}-8$, and $\mathrm{H}-8^{\prime}$ were determined to be $\alpha-, \alpha$-, and $\beta$-oriented, respectively, as de- 
duced from the ROESY correlation of $\mathrm{H}-9$ with $\mathrm{H}-8$ and the small coupling constant between $\mathrm{H}-9$ and $\mathrm{H}-8(J=1.0 \mathrm{~Hz})$.

Compounds $\mathbf{1}-\mathbf{1 0}$ were tested for cytotoxicity against HepG2, SGC7901, HL-60, and K562 cell lines in vitro. However, none of them were cytotoxic.

\section{Experimental}

General Experimental Procedures Optical rotation was measured on a Horiba SEPA-300 polarimeter. IR spectra were obtained with a Tensor 27 FT-IR spectrometer with $\mathrm{KBr}$ pellets. The ${ }^{1} \mathrm{H}-,{ }^{13} \mathrm{C}$-, and 2D-NMR spectra were recorded on Bruker AV-400 or DRX-500 spectrometers at room temperature ( $\delta$ in ppm, $J$ in $\mathrm{Hz}$ ). FAB-MS and HR-ESI-MS measurements were carried out on a VG Autospec-3000 spectrometer. Silica gel (200-300 mesh), Silica gel H (Qingdao Marine Chemical Ltd., China), and Sephadex LH-20 (Amersham Pharmacia Biotech, Sweden) were used for column chromatography. Fractions were monitored using TLC, and spots were visualized by heating silica gel plates immersed with $15 \% \mathrm{H}_{2} \mathrm{SO}_{4}$ in ethanol. Solvents were distilled before use. Semipreparative HPLC was performed on an Agilent 1100 liquid chromatograph with a Zorbax SB-C 18 (9.4 mm $\times 25 \mathrm{~cm})$.

Plant Material The aerial parts of $V$. foetidum var. foetidum were collected in Simao County, Yunnan Province, People's Republic of China, in June 2005. The plant was identified by professor Xiao Cheng, Kunming Institute of Botany, Yunnan. A voucher specimen was deposited at the Kunming Institute of Botany with identification number 20050625.

Extraction and Isolation The dried and powdered aerial parts of $V$. foetidum var. foetidum $(6.5 \mathrm{~kg})$ were extracted with $70 \%$ aqeuous $\mathrm{Me}_{2} \mathrm{CO}$ $(3 \times 351,3 \mathrm{~d}$, each) at room temperature. The extract was evaporated to dryness under reduced pressure to obtain a residue $(700 \mathrm{~g})$. The residue was suspended in water $(1000 \mathrm{ml})$ and then extracted with ethyl acetate to afford the ethyl acetate extract $(352 \mathrm{~g})$. The ethyl acetate-soluble extract was subjected to silica gel column chromatography eluted with gradient petroleum ether- $\mathrm{Me}_{2} \mathrm{CO}(100: 0 \rightarrow 0: 100)$ to yield fractions $1-10$. Fraction $3(19.2 \mathrm{~g})$ was further purified by silica gel column chromatography using petroleum ether- $\mathrm{Me}_{2} \mathrm{CO}(8: 2)$ as eluents to provide subfractions 3.1-3.5. Subfraction $3.2(4.1 \mathrm{~g})$ was subjected to Sephadex $\mathrm{LH}-20$ chromatograpy $\left(\mathrm{CHCl}_{3}-\mathrm{MeOH}\right.$ $1: 1)$ and then further purified by silica gel column chromatography eluted with $\mathrm{CHCl}_{3}-\mathrm{Me}_{2} \mathrm{CO}(20: 1)$ to yield compounds 1 (150 mg), 2 (72 mg), 5 $(15 \mathrm{mg})$, and $\mathbf{6}(8 \mathrm{mg})$. Fraction 4 (15.6 g) was separated by silica gel column chromatography eluted with petroleum ether- $\mathrm{Me}_{2} \mathrm{CO}(7: 3)$ to yield six subfractions 4.1-4.6. Subfraction $4.3(2.8 \mathrm{~g})$ was subjected to Sephadex LH-20 chromatography $\left(\mathrm{CHCl}_{3}-\mathrm{MeOH} 1: 1\right)$ and followed by semipreparative HPLC ( $\left.\mathrm{MeOH}-\mathrm{H}_{2} \mathrm{O} ; 50: 50\right)$ to provide $\mathbf{3}(7 \mathrm{mg}), \mathbf{4}(4 \mathrm{mg})$, and $\mathbf{1 0}(6.0 \mathrm{mg})$. Subfraction 4.6 was subjected to repeated silica gel column chromatography to give $7(5 \mathrm{mg}), \mathbf{8}(14 \mathrm{mg})$, and $\mathbf{9}(8 \mathrm{mg})$.

Compound (1): White amorphous powder; $[\alpha]_{\mathrm{D}}^{23.3}-64.7(c=0.1, \mathrm{MeOH})$; UV (MeOH) 282 (4.0), 231 (2.9), 204 (3.3); IR (KBr) $v_{\max } \mathrm{cm}^{-1}: 3536$, $1611,1596,1520,1463,1434,1255,1065,1032 ;{ }^{13} \mathrm{C}-\mathrm{NMR}$, see Table 1; ${ }^{1} \mathrm{H}-\mathrm{NMR}\left(500 \mathrm{MHz}, \mathrm{CD}_{3} \mathrm{OD}\right) \delta: 6.86(1 \mathrm{H}, \mathrm{d}, J=2.0, \mathrm{H}-2), 6.79(1 \mathrm{H}, \mathrm{d}, J=$ 8.0, H-5), 6.73 (1H, dd, $J=2.0,8.0, \mathrm{H}-6), 4.67(1 \mathrm{H}, \mathrm{m}, \mathrm{H}-7), 2.39$ (1H, t, $J=$ $4.5, \mathrm{H} \alpha-9), 2.80(1 \mathrm{H}, \mathrm{t}, J=4.5, \mathrm{H} \beta-9), 6.33\left(1 \mathrm{H}, \mathrm{d}, J=2.0, \mathrm{H}-2^{\prime}\right), 6.81(1 \mathrm{H}$, d, $\left.J=8.0, \mathrm{H}-5^{\prime}\right), 6.59\left(1 \mathrm{H}, \mathrm{dd}, J=2.0,8.0, \mathrm{H}-6^{\prime}\right), 2.64(1 \mathrm{H}, \mathrm{dd}, J=10.0,14.0$, $\left.\mathrm{H} \alpha-7^{\prime}\right), 2.74\left(1 \mathrm{H}, \mathrm{dd}, J=5.0,14.0, \mathrm{H} \beta-7^{\prime}\right), 2.45\left(1 \mathrm{H}, \mathrm{m}, \mathrm{H}-8^{\prime}\right), 3.97(1 \mathrm{H}$, dd, $\left.J=4.0,9.0, \mathrm{H} \beta-9^{\prime}\right), 4.03\left(1 \mathrm{H}, \mathrm{dd}, J=6.5,8.0, \mathrm{H} \beta-9^{\prime}\right), 3.80$ (3H, s, H-1"), 3.85 (3H, s, H-1"'); FAB-MS (neg.) $m / z: 357[\mathrm{M}-\mathrm{H}]^{-}$; HR-ESI-MS $m / z$ : $357.1330[\mathrm{M}-\mathrm{H}]^{-}\left(\right.$Calcd for $\left.\mathrm{C}_{20} \mathrm{H}_{21} \mathrm{O}_{6}^{-}, 357.1338\right)$.

Compound (2): White amorphous powder; $[\alpha]_{\mathrm{D}}^{23.3}-116.7(c=0.15$, $\mathrm{MeOH})$; UV (MeOH) 282 (3.7), 231 (2.8), 204 (3.2); IR (KBr) $v_{\max } \mathrm{cm}^{-1}$ : $3523,1602,1592,1516,1433,1269,1159,1070,1023 ;{ }^{13} \mathrm{C}-\mathrm{NMR}$, see Table 1 ; ${ }^{1} \mathrm{H}-\mathrm{NMR}\left(500 \mathrm{MHz}, \mathrm{CDCl}_{3}\right) \delta: 6.89(1 \mathrm{H}, \mathrm{s}, \mathrm{H}-2), 6.81(1 \mathrm{H}, \mathrm{s}, \mathrm{H}-4), 6.80$ $(1 \mathrm{H}, \mathrm{s}, \mathrm{H}-6), 4.87(1 \mathrm{H}, \mathrm{m}, \mathrm{H}-7), 3.14(1 \mathrm{H}, \mathrm{t}, J=15.0, \mathrm{H} \alpha-9), 3.28(1 \mathrm{H}, \mathrm{t}, J=$ 15.0, H $\beta-9), 6.70\left(1 \mathrm{H}, \mathrm{d}, J=2.0, \mathrm{H}-2^{\prime}\right), 6.72\left(1 \mathrm{H}, \mathrm{d}, J=10.0, \mathrm{H}-5^{\prime}\right), 6.60$ $\left(1 \mathrm{H}, \mathrm{dd}, J=2.0,10.0, \mathrm{H}-6^{\prime}\right), 2.48\left(1 \mathrm{H}, \mathrm{m}, \mathrm{H} \alpha-7^{\prime}\right), 2.85\left(1 \mathrm{H}, \mathrm{m}, \mathrm{H} \beta-7^{\prime}\right), 2.45$ $\left(1 \mathrm{H}, \mathrm{m}, \mathrm{H}-8^{\prime}\right), 3.86\left(1 \mathrm{H}, \mathrm{dd}, J=2.5,12.0, \mathrm{H} \alpha-9^{\prime}\right), 3.95(1 \mathrm{H}, \mathrm{dd}, J=5.0,12.0$, $\left.\mathrm{H} \beta-9^{\prime}\right), 3.77$ (3H, s, H-1"), 3.80 (3H, s, H-1"'); FAB-MS (neg.) $m / z: 393$ $[\mathrm{M}-\mathrm{H}]^{-}$; HR-ESI-MS $m / z: 417.1087[\mathrm{M}+\mathrm{Na}]^{+}\left(\right.$Calcd for $\mathrm{C}_{20} \mathrm{H}_{23} \mathrm{O}_{6} \mathrm{NaCl}^{+}$, 417.1080).
Compound (3): Colorless gum; $[\alpha]_{\mathrm{D}}^{23.3}-29.4(c=0.21, \mathrm{MeOH}) ; \quad \mathrm{UV}$ (MeOH) 281 (4.2), 223 (3.7), 203 (3.0); IR (KBr) $v_{\max } \mathrm{cm}^{-1}: 3417,1602$, $1514,1466,1434,1247,1131,1029 ;{ }^{13} \mathrm{C}-\mathrm{NMR}$, see Table $1 ;{ }^{1} \mathrm{H}-\mathrm{NMR}(500$ $\left.\mathrm{MHz}, \mathrm{CDCl}_{3}\right) \delta: 6.73(1 \mathrm{H}, \mathrm{d}, J=2.0, \mathrm{H}-2), 6.75(\mathrm{~d}, J=10.0, \mathrm{H}-5), 6.62(1 \mathrm{H}$, dd, $J=2.0,10.0, \mathrm{H}-6), 2.52(1 \mathrm{H}, \mathrm{m}, \mathrm{H} \alpha-7), 2.75(1 \mathrm{H}, \mathrm{m}, \mathrm{H} \beta-7), 2.43(1 \mathrm{H}$, m, H-8), $4.62(1 \mathrm{H}, \mathrm{d}, J=5.5, \mathrm{H}-9), 6.70\left(1 \mathrm{H}, \mathrm{s}, \mathrm{H}-2^{\prime}\right), 6.82(1 \mathrm{H}, \mathrm{d}, J=10.0$, H-5'), $6.68(1 \mathrm{H}, \mathrm{d}, J=10, \mathrm{H}-6), 2.46\left(1 \mathrm{H}, \mathrm{m}, \mathrm{H} \alpha-7^{\prime}\right), 2.73\left(1 \mathrm{H}, \mathrm{m}, \mathrm{H} \beta-7^{\prime}\right)$, $2.05\left(1 \mathrm{H}, \mathrm{m}, \mathrm{H}-8^{\prime}\right), 3.58\left(1 \mathrm{H}, \mathrm{t}, J=10.0, \mathrm{H} \alpha-9^{\prime}\right), 3.99(1 \mathrm{H}, \mathrm{t}, J=10.0, \mathrm{H} \beta-$ $\left.9^{\prime}\right), 3.89\left(3 \mathrm{H}, \mathrm{s}, \mathrm{H}-1^{\prime \prime}\right), 3.87$ (3H, s, H-1"'), 3.33 (3H, s, H-1"''); FAB-MS (neg.) $m / z: 373[\mathrm{M}-\mathrm{H}]^{-}$; HR-ESI-MS $m / z: 397.1631[\mathrm{M}+\mathrm{Na}]^{+}(\mathrm{Calcd}$ for $\mathrm{C}_{21} \mathrm{H}_{26} \mathrm{O}_{6} \mathrm{Na}^{+}$, 397.1627).

Compound (4): Colorless gum; $[\alpha]_{\mathrm{D}}^{23.3}+76.5(c=0.19, \mathrm{MeOH})$; UV (MeOH) 282 (4.3), 221 (3.7), 203 (3.0); IR (KBr) $v_{\max } \mathrm{cm}^{-1}: 3418,1606$, 1513, 1466, 1433, 1247, 1159, 1065, 1029; ${ }^{13} \mathrm{C}-\mathrm{NMR}$, see Table $1 ;{ }^{1} \mathrm{H}-\mathrm{NMR}$ $\left(500 \mathrm{MHz}, \mathrm{CDCl}_{3}\right) \delta: 6.57(1 \mathrm{H}, \mathrm{d}, J=2.0, \mathrm{H}-2), 6.80(1 \mathrm{H}, \mathrm{d}, J=8.5, \mathrm{H}-5)$, $6.61(1 \mathrm{H}, \mathrm{dd}, J=2.0,8.5, \mathrm{H}-6), 2.44(1 \mathrm{H}, \mathrm{dd}, J=7.5,14.0, \mathrm{H} \alpha-7), 2.66(1 \mathrm{H}$, dd, $J=7.5,14.0, \mathrm{H} \beta-7), 2.15(1 \mathrm{H}, \mathrm{m}, \mathrm{H}-8), 4.70(1 \mathrm{H}, \mathrm{d}, J=1.0, \mathrm{H}-9), 6.62$ $\left(1 \mathrm{H}, \mathrm{d}, J=2.0, \mathrm{H}-2^{\prime}\right), 6.68\left(1 \mathrm{H}, \mathrm{d}, J=8.0, \mathrm{H}-5^{\prime}\right), 6.50(1 \mathrm{H}, \mathrm{dd}, J=2.0,8.0$, H-6 $), 2.55\left(1 \mathrm{H}, \mathrm{m}, \mathrm{H} \alpha-7^{\prime}\right), 2.75\left(1 \mathrm{H}, \mathrm{m}, \mathrm{H} \beta-7^{\prime}\right), 2.15\left(1 \mathrm{H}, \mathrm{m}, \mathrm{H}-8^{\prime}\right), 3.62$ $\left(1 \mathrm{H}, \mathrm{t}, J=8.5, \mathrm{H} \alpha-9^{\prime}\right), 3.97\left(1 \mathrm{H}, \mathrm{t}, J=8.5, \mathrm{H} \beta-9^{\prime}\right), 3.86\left(3 \mathrm{H}, \mathrm{s}, \mathrm{H}-1^{\prime}\right), 3.83$ $\left(3 \mathrm{H}, \mathrm{s}, \mathrm{H}-1^{\prime \prime \prime}\right), 3.33$ (3H, s, H-1"'''); FAB-MS (neg.) $\mathrm{m} / z$ : $373[\mathrm{M}-\mathrm{H}]^{-}$; HRESI-MS $m / z$ : $397.1636[\mathrm{M}+\mathrm{Na}]^{+}\left(\right.$Calcd for $\mathrm{C}_{21} \mathrm{H}_{26} \mathrm{O}_{6} \mathrm{Na}^{+}, 397.1627$ ).

Acknowledgments This work was supported by grants from the 973 project (No. 2009CB522300), the National Natural Science Foundation of China (No. 90813004), and the Innovation Foundation of Chinese Academy of Sciences (KSCX1-YW-R-24). The authors are also indebted to the analytical group of the State Key Laboratory of Phytochemistry and Plant Resources in West China, Chinese Academy of Sciences, for the spectroscopic measurements.

\section{References}

1) Wang L. Q., Chen Y. G., Xu J. J., Liu Y., Li X. M., ZhaoY., Chem. Biodivers., 5, 1879-1899 (2008).

2) Xu B. S., Hu J. Q., Wang H. J., "Flora Reipublicae Popularis Sinicae," Vol. 72, ed. by Xu B. S., Science Press, Beijing, 1988, p. 66.

3) Xu B. S., Hu J. Q., Wang H. J., "Flora Reipublicae Popularis Sinicae," Vol. 72, ed. by Xu B. S., Science Press, Beijing, 1988, p. 56.

4) Kagawa M., Minami H., Nakahara M., Takahashi H., Takaoka S., Fukuyama Y., Phytochemistry, 47, 1101-1105 (1998).

5) Machida K., Kikuchi M., Tetrahedron Lett., 37, 4157-4159 (1996).

6) Hase T., Iwagawa T., Dave M. N., Phytochemistry, 24, 1323-1327 (1985).

7) Fukuyama Y., Minami H., Ichikawa R., Takeuchi K., Kodama M., Phytochemistry, 42, 741-744 (1996).

8) Fukuyama Y., Fujii H., Minami H., Takahashi H., Kubo M., J. Nat. Prod., 69, 1098-1100 (2006).

9) Fukuyama Y., Nakahara M., Minami H., Kodama M., Chem. Pharm. Bull., 44, 1418-1420 (1996).

10) Zhu X. D., Wang Q., Wang Y. F., Ju P., Luo S. D., Acta Bot. Yunnan, 28, 91-94 (2006).

11) Kawazu K., Agric. Biol. Chem., 44, 1367 (1980).

12) Shen Y. C., Prakash C. V. S., Wang L. T., Chien C. T., Hung M. C., J. Nat. Prod., 65, 1052-1055 (2002).

13) Fonseca S. F., Nielsen L., Ruveda E. A., Phytochemistry, 18, 17031708 (1979).

14) Abe F., Yamauchi T., Phytochemistry, 27, 575-577 (1988).

15) Achenbach H., Waibel R., Mensah A. I., Phytochemistry, 22, 749753 (1983)

16) Estevez-Braun A., Estevez-Reyes R., Gonzalez-Perez J. A., Gonzalez A. G., J. Nat. Prod., 58, 887-892 (1995).

17) Tan R. X., Jakupovic J., Jia Z. J., Planta Med., 56, 475-477 (1990).

18) Gozler B., Kivcak B., Arar G., Gozler T., Heterocycles, 39, 243-250 (1994).

19) Liang S., Shen Y. H., Tian J. M., Wu Z. J., Jin H. Z., Zhang W. D., Yan S. K., J. Nat. Prod., 71, 1902-1905 (2008). 